\title{
The Experience of the Fight Against COVID-I9 in Clinical Laboratory Departments from Chengdu, China
}

\section{Qin Wang* \\ Chenggui Liu* \\ Jian Xu \\ Peipei Song \\ Shuzhe Yang \\ Qin Zhang}

Department of Clinical Laboratory, Chengdu Women's and Children's Central Hospital, School of Medicine, University of Electronic Science and Technology of China, Chengdu, 6II73I, People's Republic of China

*These authors contributed equally to this work

\section{Correspondence: Qin Wang}

Department of Clinical Laboratory, Chengdu Women's and Children's Central Hospital, School of Medicine, University of Electronic Science and Technology of

China, No. 1617, Riyue Avenue, Qingyang

District, Chengdu, 6II73I, People's

Republic of China

Tel +862861866000-6267

Email314306221@qq.com
Objective: In order to fight against coronavirus disease 2019 (COVID-19) better and to share our experience as a reference for clinical laboratory departments.

Methods: This was a retrospective study conducted in the clinical laboratory department of Chengdu Women's and Children's Central Hospital in Chengdu, China, from April 2020 to January 2021. The number of nucleic acid and antibody testing specimens of suspected COVID-19 cases was analyzed. The key points of suspected-case sample processing and detection in the clinical laboratory department were summarized. The laboratory was directly involved in the sample processing and testing of suspected cases, the release of reports, and the transfer of specimens to the fever clinic.

Results: The number of COVID-19 nucleic acid test specimens in our laboratory ranged from 102 to 2170 per day, and the number of antibody test specimens ranged from 24 to 391 per day. There were four main considerations in the treatment and detection of suspected-case specimens in the clinical laboratory: biosafety management in clinical laboratory departments, measures to ensure the health of the staff, the eight time points for processing suspected-case samples (turn-around time), and key points for the detection of suspected case specimens.

Conclusion: The laboratory developed a protective process for COVID-19 antibody and nucleic acid detection during the pandemic. At present, the detection of COVID-19 antibodies and nucleic acids in the clinical laboratory department is orderly, and there have been no cases of laboratory infection.

Keywords: COVID-19, clinical laboratory, laboratory tests

\section{Introduction}

Coronavirus disease 2019 (COVID-19) is a new type of acute respiratory infectious disease and systemic syndrome primarily presenting clinical symptoms of dry cough, dyspnea, and fever, which in some cases $(8-15 \%$ depending on the geographical setting and individual characteristics) lead to a critical condition. ${ }^{1}$ COVID-19 has become a major global public health event since it began in 2019. ${ }^{2}$ According to WHO data, COVID-19 has already spread worldwide, with $105,394,301$ confirmed cases in more than 223 different countries, causing more than 2,302,302 confirmed deaths as of February 8, 2021 (https://www.who.int/).

Currently, the COVID-19 pandemic threatens health systems around the world, and medical doctors at the front line of the pandemic suffer from the risk of infection, pressure, and work overload, which impacts not only the well- 
being of healthcare workers but also patient safety and the function of the healthcare system. ${ }^{3}$ As the laboratory medical staff at the front line of the fight against COVID19 , we have to think about how to perform the laboratory work and how to properly handle COVID-19 specimens, regardless of whether the work is done during the outbreak or in the current, normal pandemic prevention and control environment. To ensure the orderly development of laboratory work during the pandemic and to ensure the biosafety of staff, a summary of the previous fight against the COVID-19 pandemic is needed. We proposed several key features of the fight against COVID-19 in clinical laboratory departments, focusing primarily on risk management, continuous improvement, and staff management. We have overcome all kinds of difficulties and accumulated a wealth of experience in clinical practice during the pandemic and hope to provide some reference for other laboratory staff.

\section{Methods}

This was a retrospective study conducted in the clinical laboratory department of Chengdu Women's and Children's Central Hospital in Chengdu, China from April 2020 to January 2021. This study was done in accordance with the Helsinki Declaration and was approved by the Medical Ethics Committee of Chengdu Women's and Children's Central Hospital, Chengdu, China (B2021(15), Medical Ethics Committee, CWCCH).

After the outbreak of COVID-19, the hospital became the designated COVID-19 prevention and control hospital in Chengdu, China. During the COVID-19 pandemic, our clinical laboratory formulated prevention and control measures to handle samples in a timely manner and to protect laboratory staff from infection. The laboratory also developed a protection process for COVID-19 antibody and nucleic acid detection. We reviewed the nucleic acid and antibody test specimen numbers for suspected COVID-19 cases from April 2020 to January 2021, and summarized the key points of suspected-case sample processing and detection from four aspects. According to the peopleoriented principle, these four considerations ranked in the order of biosafety management in clinical laboratory departments, measures to ensure the health of the staff, the eight time points for processing suspected-case samples (turn-around time) and detection of suspected specimens.

\section{Results}

\section{Biosafety Management in Clinical Laboratory Departments}

When testing potentially infectious specimens, laboratories must apply biosafety measures to protect medical staff and the environment. Generally, biosafety measures depend on the risk category of pathogens, the route of infection (such as aerosols, droplets or blood), the concentration of pathogens in organs and body fluids, and the stability of pathogens in the body and the environment. ${ }^{4}$ Since suspected COVID-19 specimens can be transmitted through aerosols, droplets and direct contact, biosafety measures must be strictly enforced.

First, medical staff of the clinical laboratory department were kept up to date on the latest information about COVID-19 and the relevant knowledge of virus inactivation and biosafety. ${ }^{5,6}$ Second, as laboratories were highrisk areas with direct contact with highly pathogenic sources of infection, such as blood, sputum, throat swabs, urine and feces, regional management strategies were established to protect the medical staff and other personnel in the hospital. Suppliers or other personnel who did not wear masks were prohibited from entering the laboratory, and laboratory password locks were set. Third, standard operating procedures (SOP) were established. To ensure that medical staff were well protected, COVID-19 biosafety training and protective operation exercises were conducted according to the SOP and reminded staff of biosafety details so that employees could protect each other in a conscious and orderly manner. To minimize the risk of cross-infection, on-duty staff were encouraged perform hand hygiene and wear personal protective equipment (PPE). The specific practices were as follows: more hands-free disinfectants were placed in the departments, the disinfector operated effectively for 24 hours every day, and ultraviolet radiation was used to regularly disinfect molecular biology laboratories and microbiology laboratories. Each group arranged personnel to wipe and disinfect the surfaces in the area every day. Protective clothing, hats, medical masks, goggles, gloves and shoe covers were worn before laboratory testing, suspected specimens were tested in a biosafety cabinet, and medical waste was soaked in a biosafety cabinet and then sterilized under high pressure.

During the COVID-19 pandemic, the country immediately formulated effective management measures. Sichuan Province enforced the level-1 public health emergency 
response between 24 January and 26 February 2020, then the discussion meetings in the hospital was conducted through non-contact methods such as telephone, WeChat, online (Internet), and formulated a flow chart for the detection of suspected COVID-19 specimens. Through training (mostly online video training), all the staff studied and implemented the measures conscientiously, as shown in Figure 1.

\section{Measures to Ensure the Health of Staff}

During the COVID-19 pandemic, staff were encouraged to maintain healthy diet and sleep practices and a positive attitude and avoid declines in immunity caused by irregular life or psychological pressure. Moderate exercise could be used to enhance immunity but catching cold after high-intensity activities was to be avoided. The members of the department

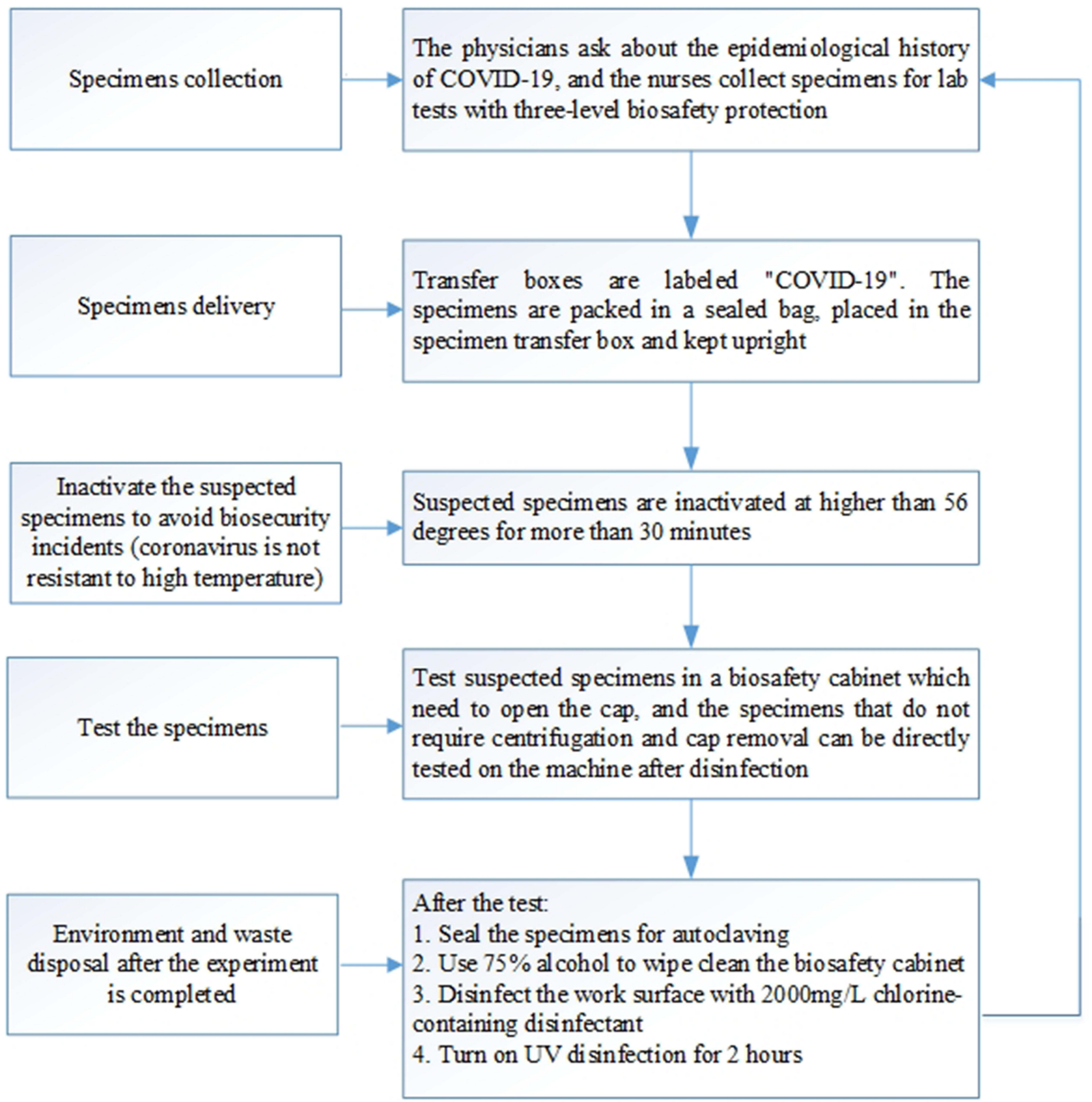

Figure I Flowchart for the detection of suspected COVID-19 specimens at Chengdu Women's and Children's Central Hospital. 
were also encouraged to reduce unnecessary gatherings. After work, the staff were to go home or return to their residence, reducing unnecessary exposure. We encouraged people to go to work by walking or cycling and reduce their use of public transport. To separate meals and keep a safe distance from employees, we temporarily adjusted the clean area space of the department for use by colleagues.

Adequate preparation of protective materials to ensure an adequate supply chain was necessary. The reagent administrator in the department strictly controlled the protective materials and should not falsely claim and use them without authorization, especially masks and protective clothing. Sterilized alcohol, chlorine-containing preparations and other sterilized products were managed according to the management requirements of hazardous chemicals. The staff conducted varying degrees of protection requirements according to the biosafety requirements of the laboratory zone, and all waste protective equipment was sterilized under high pressure.

In addition, our laboratory also implemented a series of improvement measures. To make the working environment of the staff more comfortable and protective, we built a COVID-19 nucleic acid laboratory and emergency testing laboratory. We also hired more than a dozen employees to ensure that all employees had enough rest and that if they suffered from colds or other physical diseases, they could take an appropriate amount of time off.

\section{Eight Time Points in Processing Suspected Case Samples (Turn-Around Time)}

We are a national tertiary maternal and pediatric medical healthcare organization providing the most specialized care in West China with regard to pediatric and maternal healthcare, family planning, rehabilitation, emergency treatment and training and scientific research. The average number of births in the past three years at our hospital was approximately 20,000. At the same time, we are also the leading unit of the medical federation in Chengdu that provides technology and guidance to other maternal and pediatric health institutions. Prior to the pandemic, our laboratory processed more than 5000 biological specimens per day, including secretions, blood, sputum, urine, feces and bronchoalveolar lavage (BAL). Our laboratory has completed clinical diagnostic procedures and advanced technology. The department consists of several subspecialty groups, including the Blood Group, Body Fluid Group, Biochemical Immunity Group, Infection
Immunity Group, Microorganism Group, Emergency Group, Molecular Biology Group and other branches. Our hospital has a large amount of advanced equipment that can quickly detect suspected case specimens and meet biosafety requirements, as shown in Table 1 .

Beginning on April 2020, our hospital required COVID-19 nucleic acid testing for the following people: outpatient and emergency patients with fever (examination after admission for critically ill patients), patients suspected of having novel coronavirus infection in outpatient and emergency departments, hospitalized patients with an epidemiological history or fever and respiratory symptoms, inpatients, attendants and medical staff suspected of novel coronavirus infection. Our hospital also required COVID-19 antibody testing for newly admitted children (except for newborns who had no fever or were not suspected to be infected with the novel coronavirus).

The laboratory is directly involved in the sample processing and testing of a large number of suspected cases of COVID-19, the release of reports, and the specimen transfer of suspected cases to the fever clinic. The number of COVID-19 nucleic acid test specimens in our laboratory ranged from 102 to 2170 per day. Antibody test specimens ranged from 24 to 391 per day. To cope with the increasing number of tests and ensure the accuracy and timeliness of the results, we formulated the relevant procedures and eight time points for the handling of suspected case specimens (turn-around time of $<8 \mathrm{~h}$ for non-STAT, 4-6 h for STAT, and $<2 \mathrm{~h}$ for special STAT [with no delay]). To ensure that the testing process was conducted in a timely and effective manner, eight time points of specimen turnaround time were effectively monitored: doctor application, specimen collection, specimen transfer, specimen delivery, specimen signing, specimen testing, test completion, and doctor disposal of result. After the test was completed, we also monitored air pollution, instruments, electrical appliances and garbage disposal.

\section{Key Points for the Detection of Suspected Case Specimens}

In the case of limited equipment and work areas, the laboratory strictly managed and zoned. The most important consideration was to use independent specimen transfer boxes and biosafety cabinets for the pretreatment of suspected specimens. Suspected specimens were inactivated at higher than 56 degrees for more than 30 minutes, followed by routine detection. Although some studies have 
Table I Advanced Equipment Used to Detect Specimens of Suspected Cases

\begin{tabular}{|c|c|c|c|}
\hline Devices & Manufacturer & $\begin{array}{l}\text { Functions (for Suspected Case } \\
\text { Specimens) }\end{array}$ & Characteristics \\
\hline Siemens Chemiluminescence Assembly Line & SIEMENS, Germany & $\begin{array}{c}\text { Detection of hormones and tumor } \\
\text { markers }\end{array}$ & $\begin{array}{l}\text { Fast, meet the requirements } \\
\text { of biosafety }\end{array}$ \\
\hline Hitachi 008AS Automatic Biochemistry Analyzer & HITACHI, Japan & $\begin{array}{l}\text { Detection of blood biochemical } \\
\text { indicators }\end{array}$ & $\begin{array}{l}\text { Fast, meet the requirements } \\
\text { of biosafety }\end{array}$ \\
\hline SYSMEX XT 2000I Blood Analyzer & SYSMEX, Japan & Complete blood count & $\begin{array}{l}\text { Fast, meet the requirements } \\
\text { of biosafety }\end{array}$ \\
\hline $\begin{array}{l}\text { SUCCEEDER SF8200 Automatic Coagulation } \\
\text { Analyzer }\end{array}$ & SUCCEEDER,China & Detection of coagulation function & $\begin{array}{l}\text { Fast, meet the requirements } \\
\text { of biosafety }\end{array}$ \\
\hline Beckman Coulter FC-500 Flow Cytometer & $\begin{array}{l}\text { BECKMAN } \\
\text { COULTER, USA }\end{array}$ & Immune cell classification & $\begin{array}{l}\text { Meet the requirements of } \\
\text { biosafety }\end{array}$ \\
\hline $\begin{array}{l}\text { SEBIA Capillarys } 2 \text { flex Automatic Electrophoresis } \\
\text { Apparatus }\end{array}$ & SEBIA, France & Detection of anemia & $\begin{array}{l}\text { Fast, meet the requirements } \\
\text { of biosafety }\end{array}$ \\
\hline $\begin{array}{l}\text { Merieux VI Automatic Bacteria Identification and } \\
\text { Susceptibility Analyzer }\end{array}$ & BioMerieux, France & $\begin{array}{l}\text { Bacteria identification and drug } \\
\text { sensitivity analysis }\end{array}$ & $\begin{array}{l}\text { Meet the requirements of } \\
\text { biosafety }\end{array}$ \\
\hline $\begin{array}{l}\text { Merieux BACT/ALERT 3D Automatic } \\
\text { Mycobacterium } \\
\text { Monitor System }\end{array}$ & BioMerieux, France & Blood culture & $\begin{array}{l}\text { Meet the requirements of } \\
\text { biosafety }\end{array}$ \\
\hline
\end{tabular}

found that heating can increase false negatives in experiments, ${ }^{7}$ heating is still necessary for biosafety reasons. Each experimental group worked out the standardized operation procedure for the examination of suspected specimens, and all routine tests were placed in the open laboratory. The testing focus of each team was as follows: routine blood tests and C-reactive protein were detected automatically and could not be opened, all immune specimens were collected with filter elements as much as possible, and high-risk operations involving aerosol exposure such as sputum culturing were performed in an appropriate area. The culture of secretions was completed in a safety cabinet, tables were wiped and disinfected regularly, ultraviolet light was used for disinfection of surfaces, and the laboratory prepared additional disinfection spray cans for disinfection under limited conditions, which were used to disinfect the table of the laboratory environment.

Transfer boxes were labeled "COVID-19". The specimens were packed in a sealed bag, placed in the specimen transfer box and kept upright. Before the transfer box was closed, it was disinfected with a spray such as a chlorinecontaining disinfectant or $75 \%$ ethanol, and after closure, the spray was used to disinfect the outside of the transfer box. COVID-19 specimens were transported separately and were not mixed with other items.

The laboratory transshipment personnel who completed the transfer of the specimens wore overalls, medical caps, N95 masks and gloves. When transporting specimens, the staff kept the transfer box stable and avoided severe bumpiness. They were instructed not to open the transfer box by themselves. In the event of an accident during transshipment, the transporter would dispose of the transfer box and would proceed to the receiving place to explain the situation so that both the transporter and the receiver could deal with the situation together. After being sent to the laboratory, the transfer box was placed in the biosafety cabinet under ultraviolet light for more than 30 minutes and then distributed to each professional group, in which pharynx swabs were placed in a $56{ }^{\circ} \mathrm{C}$ drying box for at least half an hour.

\section{Discussion}

China has controlled the COVID-19 pandemic through active prevention and treatment, and daily life is in order. However, as the global pandemic is still spreading and 
may exist for a long time, the risk of COVID-19 spreading in China will also persist.

As reported in the literature, the knowledge, attitude and practice of healthcare workers were needed in good results to decrease nosocomial COVID-19 infection. ${ }^{8}$ Therefore, during the COVID-19 pandemic, a series of prevention and control measures were taken in our clinical laboratory department to handle with the specimens in a timely manner and to protect laboratory staff from infection. The laboratory also developed a protection process for COVID-19 antibody and nucleic acid detection. At present, the detection of COVID-19 antibodies and nucleic acids in the clinical laboratory department is well-organized and in line with the hospital and national standards. There have been no cases of laboratory infection as of January, 2021, and the laboratory and all of its members aim to maintain the zeroinfection rate by staying vigilant and keeping abreast of the best biosafety practices as COVID-19 evolves.

In addition, the pandemic has some important implications for clinical laboratory departments. First, to address COVID-19 or other sudden public health outbreaks, we recommend that clinical laboratory departments prepare emergency test areas or COVID-19 test areas. Second, with regard to the importance of talent reserves and personnel biosafety training, new employees must undergo strict training and assessment before they can begin work and should have laboratory medicine learning at least once a month. Third, each group should make use of the network to conduct online and offline efficient training or case sharing. For the preparation of materials and consumables, hospitals should ensure the adequacy of the supply chain, especially the reserve of instruments and equipment in line with the biosafety of the laboratory and the reserve of protective materials. To cope with the possible recurrence of pandemics and the associated COVID-19 nucleic acid and antibody screening and testing, it may be necessary to keep a stock of some testing products and protective materials.

To cope with the detection of COVID-19 nucleic acid and serum antibodies in a normal large-scale population, clinical laboratory departments should expand their detection capacity, which is also a key part of the response to the current pandemic. In the course of work, if the experimental equipment cannot meet the requirements of the increasing workload, the laboratory should apply to the hospital for new instruments, equipment and materials, and protective materials will be given priority to the staff of the COVID-19 nucleic acid testing area. This is only single center experience, and it is also the limitation of the study. We hope that the results of multi-center research can be shared in the future.

\section{Ethical Approval}

This study was done in accordance with the Helsinki Declaration and was approved by the Medical Ethics Committee of Chengdu Women's and Children's Central Hospital, Chengdu, China (B2021(15), Medical Ethics Committee, $\mathrm{CWCCH}$ ). All data were anonymized to maintain participant's privacy. In light of the retrospective and anonymous nature of the study, the Ethics Committee did not require written informed consent provided by participants.

\section{Funding}

This study was supported by the Sichuan Provincial Medical Youth Innovative Scientific Research Foundation of China (Q20069), the Sichuan Provincial Science and Technology Department Research Foundation of China (2020YFS0494), and the Sichuan Provincial Science and Technology Department Research Foundation of China (2013FZ0080).

\section{Disclosure}

The authors of this manuscript have no conflicts of interest to disclose.

\section{References}

1. Xu Z, Shi L, Wang Y, et al. Pathological findings of COVID-19 associated with acute respiratory distress syndrome. Lancet Respir Med. 2020;8(4):420-422. doi:10.1016/S2213-2600(20)30076-X

2. Lu H, Stratton CW, Tang YW. The Wuhan SARS-CoV-2-what's next for China. J Med Virol. 2020;92(6):546-547.

3. Lopez-Hernandez LB, Diaz BG, Gonzalez EOZ, et al. Quality and safety in healthcare for medical students: challenges and the road ahead. Healthcare (Basel). 2020;8:4

4. Loibner M, Langner C, Regitnig P, Gorkiewicz G, Zatloukal K. Biosafety requirements for autopsies of patients with COVID-19: example of a BSL-3 autopsy facility designed for highly pathogenic agents. Pathobiology. 2021;88(1):37-45.

5. He Y, Lin Z, Tang D, Yang Y, Wang T, Yang M. Strategic plan for management of COVID-19 in paediatric haematology and oncology departments. Lancet Haematol. 2020;7(5):e359-e362.

6. World Health Organization.Available from: https://www.who.int/emer gencies/diseases/novel-coronavirus-2019). Accessed September 29, 2021.

7. Hu X, An T, Situ B, et al. Heat inactivation of serum interferes with the immunoanalysis of antibodies to SARS-CoV-2. J Clin Lab Anal. 2020;34(9):e23411.

8. Rizki SA, Kurniawan J, Budimulia P, et al. Knowledge, attitude, and practice in Indonesian health care workers regarding COVID-19. Asia Pac J Public Health. 2021;19:10105395211011017. 


\section{Publish your work in this journal}

Risk Management and Healthcare Policy is an international, peerreviewed, open access journal focusing on all aspects of public health, policy, and preventative measures to promote good health and improve morbidity and mortality in the population. The journal welcomes submitted papers covering original research, basic science, clinical \& epidemiological studies, reviews and evaluations, guidelines, expert opinion and commentary, case reports and extended reports. The manuscript management system is completely online and includes a very quick and fair peer-review system, which is all easy to use. Visit http://www.dovepress.com/testimonials.php to read real quotes from published authors.

Submit your manuscript here: https://www.dovepress.com/risk-management-and-healthcare-policy-journal 